\title{
Étude de la contamination de surface des carcasses de bovins dans la zone d'abattage de Kandi, nord du Bénin.
}

\author{
LATIFOU BELCO Akim 1,2, DRAMANE Gado 2 DJEGBE Innocents, ADEGBOLA Ahmed ${ }^{1}$, Daouda \\ OUASSOU IMOROU ${ }^{1}$ Séydou et AHYI Virgile 1 \\ 1 Laboratoire de sécurité sanitaire, d'expertises et d'analyses. Institut Régional du génie industriel des \\ biotechnologies et sciences appliquées (IRGIB Africa) 07 BP 231 Cotonou Bénin. \\ $2 \quad$ École Normale Supérieure de Natitingou, Département des Sciences de la vie et de la Terre. \\ Auteur correspondant : gdramane@gmail.com
}

Original submitted in on $5^{\text {th }}$ May 2017. Published online at www.m.elewa.org on $30^{\text {th }}$ June 2017 https://dx.doi.org/10.4314/jab.v114i1.11

\section{RÉSUMÉ}

Dans ce travail, nous avons évalué en surface la qualité bactériologique des carcasses bovines dans l'aire d'abattage de Kandi au nord du Bénin, en suivant les indications techniques spécifiés dans une note de service des services vétérinaires sur les critères microbiologiques pour les carcasses.

Pour un total de la flore mésophile aérobie de $106 \mathrm{ufc} / \mathrm{cm}^{2}$ nous avons trouvé un grand nombre de bacilles gram $(+)$ $(40,74 \%)$ et les cocci Gram (+) (38,88\%). Le Gram (-) bacilles étaient de l'ordre de 8,14\% et les champignons $12,24 \%$. Mots-clés: carcasse bovine, abattoir, microbiologie, législation.

\section{Study of surface contamination of bovine carcasses in the Kandi slaughter zone, northern Benin ABSTRACT}

In this work, we have evaluated the bacterial quality of bovine carcasses in the kandi area in northern Benin, following the technical indications specified in a memo from the veterinary services on the microbiological criteria for carcasses. .

For a total aerobic mesophilic flora of $106 \mathrm{cfu} / \mathrm{cm} 2$ we found a large number of gram (+) bacilli (40.74\%) and Gram cocci $(+)(38.88 \%)$. The Gram (-) bacilli were $8.14 \%$ and the fungi $12.24 \%$.

Keywords: Bovine carcass, slaughterhouse, microbiology, legislation

\section{INTRODUCTION}

Par sa valeur nutritive, le bœuf est un aliment de choix. L'eau, sa haute valeur biologique élevée en protéines en fait un aliment essentiel pour une alimentation équilibrée. Cependant, et précisément en raison de ses qualités nutritionnelles, la viande est un terrain fertile pour la croissance microbienne. La qualité sanitaire de la viande dépend, d'abord introduit par contamination des mains des opérateurs, les outils de travail et des plans de travail au cours de l'abattage et la découpe, et deuxièmement le développement et la croissance de la flore bactérienne pendant le refroidissement, le stockage et la distribution. Les zones d'abattage sont l'un des principaux points critiques dans l'hygiène de la viande. L'abattage est la principale phase de contamination. Ainsi, 80 à $90 \%$ de la microflore trouvés dans la viande provenant d'abattoirs (Jouve, 1990 Cartier, 2007). En effet, les différentes étapes de l'abattage comme le dépouillement et l'éviscération présente une multitude de possibilités de transférer les germes sur les carcasses. La plupart de ces germes sont saprophytes et causer 
des dommages. Ces bactéries et champignons (levures et moisissures). Les germes d'altération des caractéristiques organoleptiques de l'incidence sur les carcasses. Ils provoquent la putréfaction; c'est à dire une modification importante de la viande. Les toxi-infections et d'autres maladies infectieuses Les maladies sont souvent liées à des défauts de santé et peuvent avoir des conséquences graves (Arvieux, 1998). Parmi ces pathogènes; nous citons: Salmonella spp, Escherichia coli, Staphylococcus aureus, Listeria monocytogenes, Yersinia

\section{MATÉRIELS ET MÉTHODES}

Partie de l'étude : La zone est un abattage Kandi pers sur l'établissement, sous la tutelle du Ministère de l'Agriculture de l'élevage et de la pêche. II est situé dans le nord-est de la ville en direction de Sègbana. Construit avec des matériaux solides, le domaine de l'abattage Kandi à une surface totale de $2 \mathrm{ha}$.ll est fonctionnel depuis 1994. II a une superficie d'Housing les animaux, deux salles pour l'abattage de bovins et de moutons. L'équipement de travail (haches, couteaux) n'est pas personnalisé. L'abattage a lieu parfois en l'absence du vétérinaire. L'évacuation des déchets et des eaux usées se fait par des tuyaux en PVC pour les fosses septiques. Le laboratoire central d'application et Parakou IRGIBAfrica l'éducation est situé dans un quartier au nord de la ville. Son unité de microbiologie a réalisé l'analyse d'échantillons prélevés à l'abattage de Kandi.

L'échantillonnage des matériaux, et les analyses microbiologiques: Équipé d'une combinaison appropriée (robe, bonnet, bottes et tablier), tampons et solutions d'échantillon, l'échantillon a été pris à des points désignés. Ils prennent en compte l'ensemble de la chaîne de traitement, de sacrifice, matériels de coupe, le banc de tuyaux adjacents et le sol. L'étude a été réalisée en marbre de Kandi tuer 45 échantillons provenant de 9 carcasses bovines au cours d'une période allant $\mathrm{du}$ $21 / 09 / 2015$ au 25/09/2015. Tous les trois jours, 7 heures les différentes étapes de l'abattage ont ensuite observé les prélèvements sur les carcasses ont été effectués par écouvillonnage. En fait, l'écouvillon a été frotté, pendant au moins 20 secondes sur la surface de la carcasse délimitée verticalement, horizontalement et en diagonale. Pour tenir compte de l'hétérogénéité de la contamination de la carcasse, des échantillons ont été prélevés à partir enterocolitica, Clostridium perfringens, Bacillus cereus (FOURNAUD et Jouve, 1990). L'objectif de notre étude était d'évaluer le niveau global de la contamination de surface des carcasses de bovins d'abattage de zone Kandi dans le nord du Bénin et en particulier, à un taux de bactéries sur les carcasses bovines, montrent l'implication des manipulateurs de la contamination de la carcasse et enfin d'éduquer au respect des normes de l'hygiène des carcasses et l'abattage en vertu de Kandi.

d'une zone de10 $\mathrm{cm} 2$ à cinq différentes régions anatomiques : la jambe, l'épaule, la cuisse, le flanc et collier. Transport et stockage d'échantillons ont été effectués à l'aide d'un refroidisseur isotherme pour une période de 3 heures pour quantitativement et qualitativement, stabiliser la flore microbienne dans les écouvillons. La température était contrôlée à l'aide d'un thermomètre. Dans le laboratoire, une nouvelle nous a permis d'évaluer la présence possible de microorganismes tels que les parasites et les champignons. La coloration de Gram a été effectuée sur la propagation et les frottis bactérien fixe. Le violet de gentiane carbolic utilisé à la couleur pour l'incubation d'une minute suivie d'un petit jet d'eau de rinçage. Le frottis bactérien est couvert de l'hydrogène pendant une minute puis égouttés et rincés à fond. Pendant une minute, l'alcool $95^{\circ}$ fade les bactéries à gram négatif est alors rincé. Frottis bactérien est couvert de safranine ou de fuchsine phéniquée pendant une minute puis la préparation était lavé une séché. Une observation de la préparation sous un microscope optique à $100 \mathrm{X}$ objectif avec huile à immersion permet de remarquer les bactéries gram $(+)$ en violet et rose Gram (-). Des échantillons ont été ensemencées sur milieu CLED d'atteindre un taux de bactéries, et moyen de l'OGE pour isoler les bactéries mésophiles aérobies, les bacilles gram négatif véhiculé par la flore normale de l'animal et l'eau de rinçage de la magistrature. Le milieu Chapman pendant ce temps peut isoler Staphylococcus aureus. Les boîtes de Pétri inoculés sont incubées dans un four pendant 18 à $24 \mathrm{~h}$ à $37^{\circ} \mathrm{C}$. La lecture des boîtes est d'évaluer la charge bactérienne, physiques et biochimiques, et formation des colonies. 
Latifou et al., J. Appl. Biosci. 2017 Étude de la contamination de surface des carcasses de bovins dans la zone d'abattage de Kandi, Nord du Bénin.

RÉSULTATS

Tableau 1 : Moyenne des analyses bactériologiques des 3 Jours de prélèvement sur (05) sites

\begin{tabular}{|l|l|l|l|l|l|l|l|}
\hline \multicolumn{2}{|c|}{ SITES } & Gigot & Flanc & Encolure & Cuisse & Épaule & Paillasse \\
\hline $\begin{array}{l}\text { PARAMÈTRES } \\
\text { Flore aérobie mésophile totale }\end{array}$ & $1,1.10^{6}$ & $1,3.10^{6}$ & $10^{6}$ & $1,2.10^{6}$ & $10^{6}$ & $1.6 .10^{6}$ \\
\hline $\begin{array}{l}\text { BFC/cm2) } \\
(+)\end{array}$ & $\begin{array}{l}\text { Coryne } \\
\text { bacteriumdiphteria }\end{array}$ & $\begin{array}{l}55 \% \\
(p=0,04)\end{array}$ & $\begin{array}{l}88 \% \\
(p=0,01)\end{array}$ & $\begin{array}{l}44 \% \\
(p=0,04)\end{array}$ & $\begin{array}{l}44 \% \\
(p=0,05)\end{array}$ & $\begin{array}{l}44 \% \\
(p=0,05)\end{array}$ & \\
\hline Bacilles Gram (-) & Vibrions & & & & & & $\begin{array}{l}11 \% \\
(p=0,04)\end{array}$ \\
\cline { 2 - 8 } & $\begin{array}{l}\text { Entérobactéries } \\
(E \text { coli) }\end{array}$ & $\begin{array}{l}11 \% \\
(p<0,01)\end{array}$ & & & $\begin{array}{l}11 \% \\
(p<0,01)\end{array}$ & & $\begin{array}{l}11 \% \\
(p<0,01)\end{array}$ \\
\hline Cocci Gram (+) & $\begin{array}{l}\text { Staphylococcus } \\
\text { aureus }\end{array}$ & $\begin{array}{l}33 \% \\
(p=0,08)\end{array}$ & $\begin{array}{l}22 \% \\
(p=0,04)\end{array}$ & $\begin{array}{l}100 \% \\
(p=0,07)\end{array}$ & $\begin{array}{l}55 \% \\
(p=0,04)\end{array}$ & $\begin{array}{l}88 \% \\
(p=0,05)\end{array}$ \\
\hline Champignons & & $\begin{array}{l}11 \% \\
(p=0,05)\end{array}$ & $\begin{array}{l}11 \% \\
(p=0,05)\end{array}$ & $\begin{array}{l}22 \% \\
(p=0,05)\end{array}$ & $\begin{array}{l}22 \% \\
(p=0,05)\end{array}$ & \\
\hline
\end{tabular}

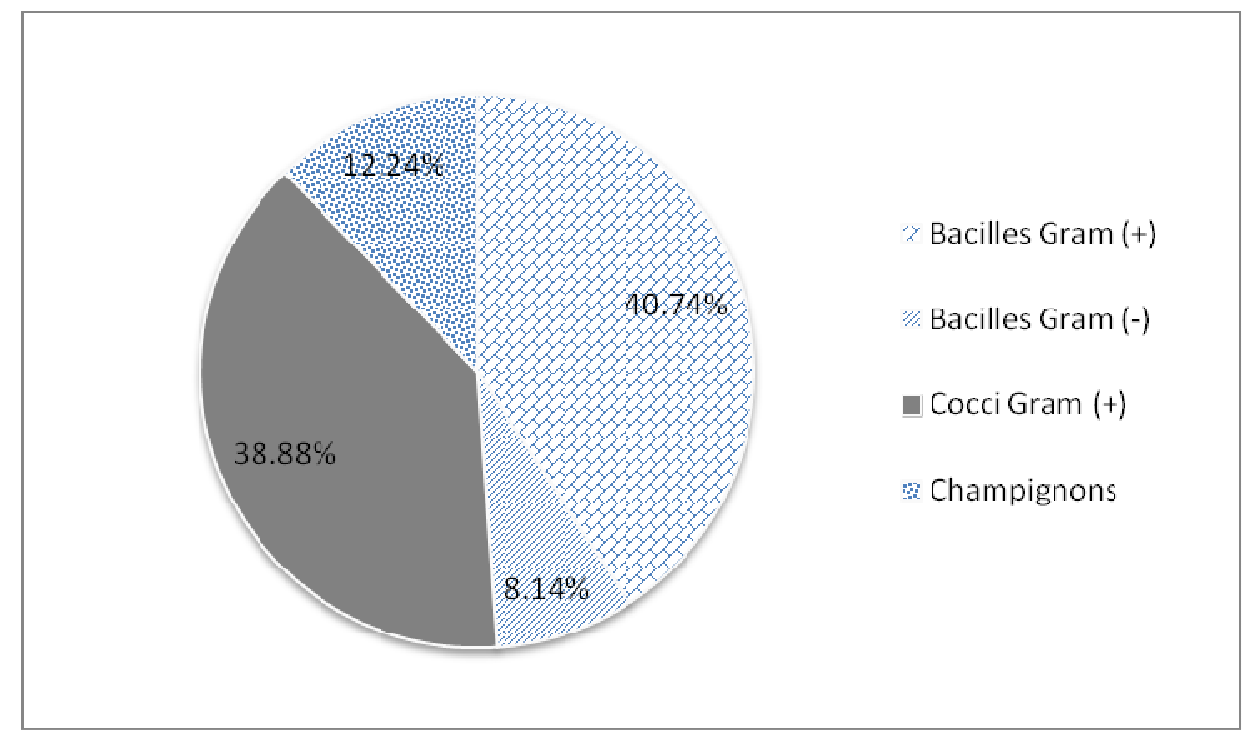

Figure 1 : Répartition des micro-organismes trouvés sur les carcasses bovines dans la région de Kandi abattage

\section{DISCUSSION}

Se référant à l'Ordonnance 72-31 (Jord, 1985) sur la sécurité des animaux et de l'inspection des aliments d'origine animale, le décret 85-243 (JORPD, 1985) et le règlement $(C E) n^{\circ} 853 / 2004$ du Parlement européen et du Conseil du 29 avril 2004 fixant des règles spécifiques d'hygiène applicables aux denrées alimentaires sur la production et la commercialisation des denrées alimentaires, les aliments, en particulier d'origine animale doivent se conformer aux exigences juridiques concernant les contaminants, substances indésirables et les critères microbiologiques. Le vétérinaire veille au respect des spécifications. Au Bénin, il n'y a pas de règlements qui précisent les critères microbiologiques. Se référant à la législation européenne, le suivi de l'hygiène générale de l'abattoir de la production est un must. II est basé sur l'établissement de procédures élaborées en conformité avec les principes HACCP et comprend en particulier les contrôles microbiologiques concernant des produits et des outils de production. Dans le cadre de notre travail, nous avons évalué la qualité bactériologique des carcasses de bovins dans la région de Kandi Slaughter air après les prescriptions techniques précisées dans un protocole des services vétérinaires, sur les critères microbiologiques pour les carcasses. La flore 
mésophile aérobie totale, par rapport à la période d'échantillonnage, la moyenne de la charge microbienne a été de $6,0 \mathrm{log}$ ufc/cm2. Exprimé en log UFC/g ou log $\mathrm{UFC} / \mathrm{cm}^{2}$, la valeur moyenne trouvée dans cette étude montrent dans l'ensemble un assez haut degré de contamination de l'échantillon de carcasses. C'est légèrement plus élevée que dans l'abattoir municipal de Kénitra au Maroc sur 32 carcasses échantillonnées (5,15 $\log u f c / g$ ) Dennaï et al. (2001) et inférieure à celle enregistrée par Oumokhtar et al. (1998) sur 20 échantillons de viande de l'abattoir Rabat $(8,10 \mathrm{~g} /$ l'UFC). Le travail de Salifou et al. (2012) relative à l'hygiène de l'abattage dans les abattoirs de Cotonou et Porto Novo) a donné une moyenne de 3,0 $\pm 0,12$ biocontamination log UFC/ $/ \mathrm{cm}^{2}$ Novembre-décembre 2009 et 5,09 $016 \mathrm{log}$ UFC/ $/ \mathrm{cm}^{2}$ Novembre-décembre 2010 pour la flore aérobie mésophile. Les résultats obtenus par Salifou et al. (2012) sont inférieurs à ceux obtenus dans cette étude. Conformément au règlement no 2073/2005 de l'Union européenne (Commission européenne, 2005), ces résultats ne sont pas satisfaisants et mauvaise hygiène des carcasses de bovins et de locaux. La forte charge FMAT observé à l'abattoir, indique à la fois une carcasse d'hygiène général défectueux en cas de non-conformité et d'autre part l'efficacité des mesures sanitaires jugées insatisfaisantes dans l'abattoir. Conformément au règlement no 2073/2005 de l'Union européenne (Commission européenne, 2005), ces résultats ne sont pas satisfaisants et mauvaise hygiène des carcasses de bovins et de locaux. La forte charge FMAT observé à l'abattoir, indique à la fois une carcasse d'hygiène général défectueux en cas de non-conformité et d'autre part

\section{CONCLUSION}

Ces résultats sont la conséquence, d'abord de l'insuffisance des installations et des équipements, y compris l'absence d'un local frigorifique et d'une chambre froide, et deuxièmement, des faiblesses identifiées dans le domaine de l'hygiène et l'exploitation de la zone d'abattage. Bien que le contrôle macroscopique des carcasses soit convenablement effectué par un contrôleur des produits d'équipe, nos résultats montrent que le problème de la qualité microbiologique des carcasses de bœuf à partir de la salle d'abattage Kandi reste non résolu. L'abattoir constituant l'un des principaux points critiques sur la qualité hygiénique de la viande en avion, il est impératif de minimiser la contamination microbienne par apporter des améliorations en matière d'hygiène, d'installations, d'équipement, le fonctionnement et le comportement personnel. D'autre part, il y aurait probablement une multiplication des micro-organismes et l'efficacité des mesures sanitaires jugées insatisfaisantes dans l'abattoir. Conformément au règlement no 2073/2005 de l'Union européenne (Commission européenne, 2005), ces résultats ne sont pas satisfaisants et mauvaise hygiène des carcasses de bovins et de locaux. La forte charge FMAT observé à l'abattoir, indique à la fois une carcasse d'hygiène général défectueux en cas de non-conformité et d'autre part l'efficacité des mesures sanitaires jugées insatisfaisantes dans l'abattoir. Total pour la flore mésophile aérobie de $106 \mathrm{CFU} / \mathrm{cm} 2$ nous avons compté un grand nombre de bacilles gram $(+)(40,74 \%)$ et les cocci Gram (+) $(38,88$ $\%$ ). Bacilles gram (-) sont de l'ordre de $8,14 \%$ et $12,24 \%$ des champignons. Cette forte représentation des bacilles gram (+) indique la présence de Corynebacterium diphtéria qui signale une contamination endogène de la carcasse de bœuf. Les cocci Gram (+) sont représentés par Staphylococcus aureus et signale une contamination exogène en raison de la non-conformité aux conditions de l'abattage. En effet, la carcasse est peut être contaminé par la peau de l'animal même, le corps du manipulateur, y compris une panne de vidange qui est une étape cruciale pour assurer sa sécurité en germe. En outre, l'équipement de travail tel que des haches, des machettes et taille-crayons sont également une source de contamination (Collobert, 2002 et 2007). Les entérobactéries et les champignons et les contenus stomacaux sont également remarqués et peuvent renforcer notre hypothèse selon laquelle les surfaces endogènes et exogènes sont une source de contamination des carcasses.

probablement une recontamination dans le circuit à l'extérieur de la zone d'abattage (chargement, transport, déchargement, découpe, transformation, réfrigération). Nous notons l'absence presque totale de la législation, y compris des lois, règlements, exigences et procédures qui garantissent la protection de la santé humaine (et animale), la protection des droits des consommateurs et le commerce dans de bonnes conditions. II est nécessaire d'établir une structure institutionnelle pour assurer la liaison avec les organisations non gouvernementales et le secteur privé et faciliter également la contribution des différents professionnels tels que les vétérinaires, les agents de contrôle des produits d'origine animale, la production animale. Les autorités pourraient mettre en place un système d'assurance qualité par l'établissement de normes de sécurité de la santé sur les dangers de santé physique, biologique ou chimique, et d'assurer la 
conformité aux exigences réglementaires de manière systématique, une vérification indépendante du

\section{RÉFÉRENCES}

Cartier $P$; 2007. Mise à jour sur la qualité des carcasses et de la viande de bovins adultes Rapport Final $n^{\circ} 17$ Mai 32022 Ministère de la qualité de la viande, Département Technique et la qualité de reproduction, pp 12-58.

Collobert JF F. Dorey, Dieuleveux C. Maele GV. 2002. qualité bactériologique de surface des carcasses de bovins. Food Science, 22 (3) 327-334

Collobert F. Dorey, Dieuleveux C. Maele GV ; 2007. Évaluation de l'efficacité du nettoyage et de la désinfection d'un atelier de découpe de viande bovine. sciences de l'alimentation ; 27 (1) 47-57

Kharrati Dennaï N., B., El Yachioui M ; 2001. Évaluation de la qualité microbiologique des carcasses de bovins fraîchement abattu. Ann. Med. L'EFP. $145,270-274$. programme et vérification.

La Direction générale de l'alimentation (2013); modification du mémo EB / SDSSA / N 20078275 sur les critères microbiologiques applicables aux carcasses d'animaux de boucherie et de volailles, et des lignes directrices sur des surfaces de contrôle du matériel et de l'abattoir de découpe d'animaux de boucherie et de volaille.

Salifou Dahouda millions CFA Ahounou GS, SK Kassa, Tugan PU Farougou S, Mensah GA, Salifou S Clinquart UN Youssao AKI, 2015. Processus d'abattage de bétail vers les abattoirs d'évaluation / Porto Novo Cotonou Bénin du sud, revue internationale de sciences biologiques et chimiques. 6 (6) : 6049-6061 